\title{
Rapid Measles Exposure Assessment in an Urban Emergency Department Using a Syndromic Surveillance System
}

\author{
Christopher Sikora*1, 2 , Kerri Fournier ${ }^{1}$, Hussain Usman ${ }^{1}$, Angela Jacobs ${ }^{1}$, Bryan \\ Wicentowich ${ }^{1}$ and James Talbot ${ }^{3,2}$
}

${ }^{1}$ Alberta Health Services, Edmonton, AB, Canada; ${ }^{2}$ University of Alberta, Edmonton, AB, Canada; ${ }^{3}$ Alberta Health, Edmonton, $A B$,

Canada

\section{Objective}

Following a clinical case of measles presenting to an urban emergency department (ED), the local health authority sought to identify all patients that might be at risk for disease. This list of contacts was generated through a manual search of hospital records and through a piloted automated data query of the health authority's syndromic surveillance system, Alberta Real Time Syndromic Surveillance Net (ARTSSN). The purpose of this pilot study was to: 1) compare the completeness of the two lookup methods and, 2) describe the time requirements needed for each method.

\section{Introduction}

In November of 2011, the local Public Health unit responsible for the Edmonton area (population 1.2mil) was alerted to an individual meeting the case definition for measles in the ED. A key part of the management strategy was to identify contacts to the index case, perform a risk assessment and, if applicable, inform them of the risk. Given the transmission characteristics, the risk for this group was defined as those present within the geographic area/environment of the index case within a specified time period. Public Health utilized the established manual lookup of hospital records and piloted an automated data query through the syndromic surveillance system, ARTSSN. This served as opportunity to validate the ability to generate a contact list, based on risk geography and time, of the ARTSSN system, and to compare the timeliness of each result.

\section{Methods}

Staff at the ED manually identified at-risk individuals who were within the ED during the risk time period. The risk period was defined by: the time the index patient arrived in the department, until two hours after the patient's departure. ARTSSN was used to identify individual-level health information from the ATD (admission-transfer-discharge) data feed of the ED operational system. This time feed measures patients' arrival (registration) and disposition (departure) within the ED. The public risk groups were therefore defined as those who:

1. Arrived within the risk period and departed after the risk period

2. Arrived before the risk period and departed within the risk period

3. Arrived AND departed within the risk period

4. Arrived before the risk period and departed after the risk period

Unique patient identifier, from each, was inputted into Microsoft Excel for comparative purposes to the ARTSSN list.

\section{Results}

The ARTSSN automated patient list was successful in identifying a total of 137 contacts compared to 108 from the manual method. This presents an overall $27 \%$ improvement in the generation of an ED contact list utilizing the registration and discharge time-stamp data from the syndromic surveillance system. All 108 cases identified by the manual recovery method were identified in the ARTSSN patient list. ARTSSN outperformed the manual method in all risk groups (32 vs. 20,53 vs. 44,48 vs. 41 and 4 vs. 3 for groups 1-4, respectively). The manual lookup method required over 8 hours of sorting and transcription by a specialist nurse compared to less than one hour of analyst time through ARTSSN.

\section{Conclusions}

This time-based risk assessment demonstration was successful in identifying individuals geographically present within an urban ED during a defined time period. The ARTSSN system, which collects time stamp arrival and departure data, and was able to capture $27 \%$ more valid contacts; a significant ncrease over the current manual operational protocol.

The ARTSSN methodology was significant in demonstrating the ability to capture patient-specific information as defined by risk time periods. The data request was defined, developed and completed in a rapid time period utilizing established means of communication and Public Health investigation methodology. This demonstrates the ability of ARTSSN to be used in times where a rapid turnaround is vital to providing, for instance, post exposure investigation and management. This allows for targeted efforts and focuses population-health measures; freeing resources for other vital functions.

Such time-based risk assessment can be useful in several scenarios. As demonstrated, it is immediately useful for individual patient management for communicable diseases. Other communicable diseases that may be applicable could include: measles (as presented), varicella and tuberculosis. Also appropriate may be scenarios where time-based exposure is related to a fixed geographic environment, such as chemical, radiological or biological exposures.

\section{Keywords}

Syndromic Surveillance; Outbreak Management; Automation; Communicable Disease Control

\section{${ }^{*}$ Christopher Sikora}

E-mail: christopher.sikora@albertahealthservices.ca 